\title{
Learning to Control Dynamic Systems with Automatic Quantization
}

\author{
Charles X. Ling* Ralph Buchal ${ }^{\dagger}$
}

\begin{abstract}
Reinforcement learning is often used in learning to control dynamic systems, which are described by quantitative state variables. Most previous work that learns qualitative (symbolic) control rules cannot construct symbols themselves. That is, a correct partition of the state variables, or a correct set of qualitative symbols, is given to the learning program.

We do not make this assumption in our work of learning to control dynamic systems. The learning task is divided into two phases. The first phase is to extract symbols from quantitative inputs. This process is also commonly called quantization. The second phase is to evaluate the symbols obtained in the first phase and to induce the best possible symbolic rules based on those symbols. These two phases interact with each other and thus make the whole learning task very difficult. We demonstrate that onr new method, called STAQ (Set Training with Automatic Quantization), can aggressively partition the input variables to a finer resolution until the correct control rules based on these partitions (symbols) are learned. In particular, we use STAQ to solve the wellknown cart-pole balancing problem.
\end{abstract}

\section{Introduction}

In a dynamic and interactive environment, an agent (or robot) receives inputs from the environment via sensors, makes decisions, and transmits outputs to the environment via effectors, in order to achieve a certain goal. If the dynamic behavior is known completely or partially to the agent, traditional control methods (such as PID control, model identification) may be used to determine the appropriate control rules. However, in unknown dynamic systems (such as exploration in a new environment), or in very complicated dynamic systems (such as temperature control in a building, an economics system, weather etc.) whose behaviour is completely unknown or filled with noise, traditional control methods cannot solve the problem effectively. If the agent is adaptive, it can learn control rules gradually based on past experience, like

*Department of Computer Science, University of Western Ontario, London, Ontario, Canada N6A 5B7. Email: ling@csd.uwo.ca

${ }^{\dagger}$ Department of Mechanical Engineering, University of Western Ontario, London, Ontario, Canada N6A 5B7. 
intelligent human beings. That is, the goal of the agent can be achieved by acquiring a set of control rules without identifying the dynamic equations of the system. Thus, the learning is model free. Automating the acquisition of control rules for general dynamic systems requires a robust and reliable learning mechanism, and therefore poses a major challenge for machine learning.

Interactive learning in a dynamic environment with weak feedback on how the goal is achieved is often called reinforcement learning. From the machine learning point of view, reinforcement learning is usually regarded as unsupervised learning, because no external teacher is available to guide the system completely for every action the system takes. The system only receives weak feedback indicating the status of the system, and the feedback from the actions is often delayed or cumulative. The agent's learning task is to construct a function (called a policy or a strategy), mapping from the current state and the current sensory inputs to actions so as to maximize the discounted cumulative reinforcements or goal [Sutton, 1984; Watkins, 1989; Lin, 1990].

Model-free learning to control dynamic systems is conceptually possible. Since control rules based on dynamic equations can be expanded to a set of rules in the form of "if situation then action", model free learning acquires this set of rules directly. Although the system is described at any point in time quantitatively by a set of real numbers, usually the state-space can be grouped into several regions, and it suffices to apply a singular action in each region (situation) to achieve the goal. Therefore, model-free learning includes two subtasks: one is to group the state-space into regions appropriately, and the second is to learn the appropriate control rules (actions) for all regions.

We introduce a new algorithm called STAQ, an acronym for Set Training with Automatic Quantization, that learns qualitative rules via automatic quantization. The STAQ algorithm is applied to the well-known pole-balancing task. We show that STAQ is general and robust since the learned control rules can balance the cart-pole for an extended period of time starting from any random position. STAQ is applicable to other dynamic systems of the same class: that is, any dynamic system. with a finite number of real number sensory inputs, a finite number of output effectors, and delayed feedback. In contrast with previous methods which use predefined partitions of the state-space (fixed bias), the STAQ program employs a technique called the progressive splitting algorithm (PSA) to partition the state space dynamically, starting from a a very coarse partition, until enough resolution is reached and a set of control rules achieving the goal is learned.

\section{The Pole-Balancing Problem}

Cart-pole balancing is a very typical dynamic system. Learning to balance the cartpole was the subject of one of the earliest experiments done in machine learning, and is the learning task studied in this paper. In the definition, "A rigid pole is hinged to a cart, which is free to move within the limits of a track. The learning system attempts to keep the pole balanced and the cart within its limits by applying a force of fixed magnitude to the cart, either to the left or to the right." [Selfridge et al., 
1985] The goal is to balance the cart-pole for a certain period of time. Failure is reported when the angle of the pole or the position of the cart exceeds certain limits defined by the users. In the cart-pole system, we define that the control strategy fails to balance the cart-pole if $|x|>2.4$ meters (the cart bumps against the ends of the track) or $|\theta| \geq 12^{\circ}$. All non-failure states are treated equally well. Thus, stability, for example, is not included as part of the goal. Failure is the only feedback received by the learner. This means that the learning program is given a very weak guidance. A bad action may cause failure long after the action is applied. Thus, the evaluation of the decisions (credit assignment) can be a long process requiring many trials.

The learning algorithm is model free: it uses the simulator merely as a black box and refrains from using any knowledge embedded in the simulator. No qualitative model of the system, even the symmetric property, is available to the learning program. The only information the learner receives is the vectors of these four real number sensory inputs $(x, \dot{x}, \theta, \dot{\theta})$ at every sampling, and the failure signal when the system fails.

The pole-balancing problem has been chosen as a model for machine learning task by many researchers. It was first studied by Michie and Chambers [Michie and Chambers, 1968] in 1968, and more recently by Anderson, Barto, Selfridge, Sutton, Sammut and so on (cf [Anderson, 1986; Barto et al., 1983; Selfridge et al., 1985; Sammut and Cribb, 1990]). However, almost all of the previous work which learns the symbolic control rules requires pre-partitions of the state-space.

\section{The STAQ Algorithm}

Since the task includes both quantization and induction of symbolic rules, the learning algorithm is divided into two phases. The first phase creates better symbols based on the suggestions of inductive learning and evaluation of bias in the second phase. More specifically, the first phase extracts symbols, or partitions the real number state variables into ranges. The problem to be solved is how many ranges are sufficient for each variable and what are the end points of the ranges. The second phase is an inductive learning process that induces the best possible rules based on the current set of symbols. The learning process also evaluates the symbols constructed in the first phase. If the best symbolic rules learned cannot satisfy the success criterion, refinement of certain partitions is needed. Suggestions on how and where refinements of partitions are needed are also proposed in the learning process. The STAQ algorithm then returns to the first phase, in which these ranges are split into two - a refined set of symbols is constructed. Starting with a very coarse partition, these two phases iterate until enough resolution of state variables is obtained such that rules based on these symbols are learned to pass the success criterion.

A new algorithm, called set training is designed for inductive learning of robust rules based on a given partition. An evaluation of the current partition, i.e. halting criterion, is conducted during set training. The halting criterion is true if the best set of control rules has been found. If the best results so far still do not pass the success criterion, PSA (Progressive Splitting Algorithm) partitions the state space further based on the suggestion (high flip count) given in the evaluation. The success criterion for the learning task is that the average balancing time for 12 initial cases 
(see later) is greater than 10,000 time steps.

The STAQ algorithm is outlined in Figure 1.

\section{Read 12 Initial Cases}

\section{L1: repeat}

Apply Set Training Algorithm

$\left\{{ }^{*}\right.$ To induce the best rules based on current partition* $\}$

until (Halting Criterion = true)

if Best Average Balancing Time $<10,000$ )

then Apply Progressive Splitting Algorithm. goto L1

else Succeed!

Perform 100 tests with random initial positions

Figure 1: The STAQ Algorithm

\section{Results of the STAQ}

Using the standard parameters of the cart-pole, STAQ splits 6 times before it learns a control strategy that produces an average balancing time over 10,000 steps. Figure 2 shows the learning curve after the $4 \mathrm{th}, 5 \mathrm{th}$, and 6 th splits. As expected, before the 6th split the partitions obtained do not provide with enough resolution (symbols) for representing/learning good control rules, so the performance during set training is very poor. After the 6th split however, enough resolution is achieved, good control rules are learned, and the success criterion is satisfied. Notice that the fluctuation still exists since set training is non-monotonic, and all the boxes are coupled. The result is summarised in Table 1 . As shown in this table (and other tables), very good and robust results are produced in the 100 random tests whose initial positions are drawn randomly from $|x|<2.4$ and $|\theta|<12^{\circ}$. The balancing time of the random test is obtained using the set of boxes when maximum average balancing time is reached.

It is interesting to examine the partitions obtained by STAQ. The algorithm actually produces fewer number of boxes than the original BOXES (144 versus 162) as shown in Table 1. Observe that the acquired partition is not symmetric. The learning program has no knowledge of the symmetry property of the cart-pole system. In fact, symmetric partition is not needed to achieve the goal: one could balance the cart-pole within one side of the track only.

\subsection{Evaluation of STAQ}

To determine how well the STAQ programs could cope with altered conditions, the simulation parameters are changed. Again, STAQ is not informed of any knowledge about the dynamic system, except the vectors of four real number state variables at 
ASTA: Resetting (Runs Vs. Average)

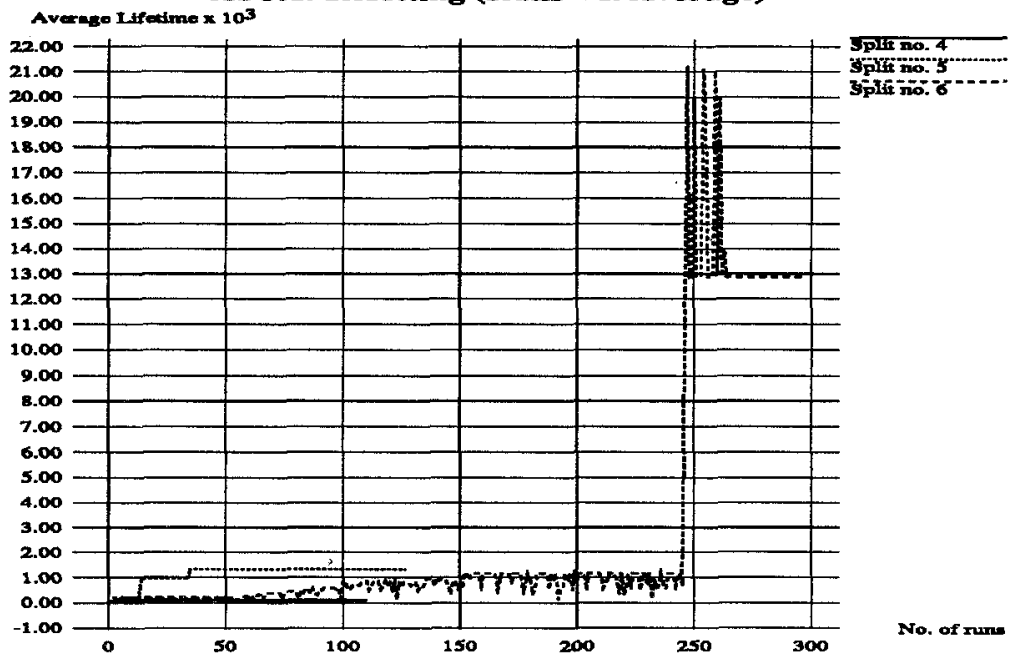

Figure 2: STAQ: Learning Curve after 4th, 5th, 6th Splitting.

sampling. This tests if STAQ can develop different sets of qualitative symbols for different cart-pole systems. Since STAQ does not take any knowledge of the dynamic system, this also tests if STAQ is general and powerful for learning to control general dynamic systems.

Variations of the simulator include reducing the force pushing right to 5 Newtons, increasing the length of the pole to $1.5 \mathrm{~m}$, and changing all the parameters except the pole length. STAQ is able to develop different partitions in all of these new environments. To summarize, variations in dynamic systems can be well sustained by the STAQ algorithm without any significant changes in results, except different qualitative symbols are constructed. Thus, STAQ is able to construct both symbols and symbolic control rules for general dynamic systems. STAQ realizes the automatic transformation from quantitative representation to qualitative representation.

\section{Conclusions}

Learning in an unknown dynamic and interactive environment with weak feedback can be very difficult. Since the sensory inputs are quantitative, both symbol construction, symbolic rule learning, and symbol evaluation are parts of the learning task. We have demonstrated that our learning algorithm STAQ accomplishes these tasks for the general dynamic systems.

\section{References}

[Anderson, 1986] Charles W. Anderson. Learning and problem solving with multilayer con- 


\begin{tabular}{||c||c|c|c||}
\hline STAQ & $\begin{array}{c}\text { Maximum average } \\
\text { of } 12 \text { initial cases }\end{array}$ & $\begin{array}{c}\text { Maximum lifetime } \\
\text { of individuals }\end{array}$ & $\begin{array}{c}\text { Average lifetime } \\
\text { of } 100 \text { random tests }\end{array}$ \\
\hline After split no. & 6 & 6 & \multirow{2}{*}{$17 \mathrm{k}$} \\
\hline After No. of runs & 247 & 247 & \\
\cline { 1 - 3 } $\begin{array}{c}\text { Balancing time } \\
\text { (in time steps) }\end{array}$ & $21 \mathrm{k}$ & $>100 \mathrm{k}$ & \\
\hline \hline $\begin{array}{c}\text { State } \\
\text { variables }\end{array}$ & \multicolumn{2}{|c||}{ Partitions } \\
\hline$x$ & \multicolumn{2}{|c||}{ (New ones inserted by SPA shown bold) } \\
\hline$\dot{x}$ & \multicolumn{2}{|c|}{$-2.40 \ldots 0.00 \ldots 1.20 \ldots 2.40$} \\
\hline$\theta$ & $-6.00 \ldots-3.00 \ldots-1.50 \ldots 0.00 \ldots 6.00$ \\
\hline$\dot{\theta}$ & $-0.20 \ldots 0.00 \ldots 0.10 \ldots 0.20$ \\
\hline
\end{tabular}

Table 1: Results of STAQ with the Standard Simulator

nectionist systems. PhD thesis, University of Massachusetts, Amherst, 1986.

[Barto et al., 1983] Andrew G. Barto, Richard S. Sutton, and Charles W. Anderson. Neuron-like elements that can solve difficult learning control problems. IEEE Trans. on Systems, Man, and Cybernetics, SMC-13(5):834-846, 1983.

[Lin, 1990] Long-Ji Lin. Self-improving reactive agents: Case studies of reinforcement learning frameworks. In Proceedings of the First International Conference on the Simulation of Adaptive Behavior, September 1990.

[Michie and Chambers, 1968] D. Michie and R. Chambers. Boxes: An experiment in adaptive control. In Machine Intelligence 2 (E. Dale and D. Michie, Eds.), pages 137-152. Oliver and Boyd, Edinburgh, 1968.

[Sammut and Cribb, 1990] Claude Sammut and James Cribb. Is learning rate a good performance criterion for learning. In Proceedings of the Seventh International Workshop on Machine Learning. Morgan Kaufmann, 1990.

[Selfridge et al., 1985] Selfridge, Richard Sutton, and Andrew Barto. Training and tracking in roboltics. In Proceedings of the Ninth International Joint Conference on Artificial Intelligence, Los Angeles, CA, 1985.

[Sutton, 1984] Richard S. Sutton. Temporal Credit Assignment In Reinforcement Learning. PhD thesis, University of Massachusetts at Amherst, 1984. (Also COINS Tech Report 84-02).

[Watkins, 1989] Chris Watkins. Learning from delayed rewards. PhD thesis, Cambridge University, 1989. 